\title{
Non-invasive brain stimulation in neglect rehabilitation: an update
}

\author{
René Martin Müri ${ }^{1,2}{ }^{*}$, Dario Cazzoli ${ }^{3}$, Tobias Nef ${ }^{2}$, Urs P. Mosimann ${ }^{2}$, Simone Hopfner ${ }^{1}$ and \\ Thomas Nyffeler ${ }^{4}$
}

1 Division of Cognitive and Restorative Neurology, Departments of Neurology and Clinical Research, Inselspital, Bern University Hospital, and University of Bern, Bern, Switzerland

${ }^{2}$ Gerontechnology and Rehabilitation Research Group, ARTORG Center for Biomedical Engineering Research, University of Bern, Bern, Switzerland

${ }^{3}$ Nuffield Department of Clinical Neurosciences, University of Oxford, Oxford, UK

${ }^{4}$ Department of Internal Medicine, Center of Neurology and Neurorehabilitation, Luzerner Kantonsspital, Luzern, Switzerland

\section{Edited by:}

Tanja Nijboer, Utrecht University, Netherlands

Reviewed by:

Alexander T. Sack, Maastricht

University, Netherlands

Giacomo Koch, Santa Lucia IRCCS,

Italy

Maike D. Hesse, University Hospital

Cologne, Germany

*Correspondence:

René Martin Müri, Division of Cognitive and Restorative Neurology,

Inselspital, 3010 Bern, Switzerland

e-mail: rene.mueri@insel.ch
Here, we review the effects of non-invasive brain stimulation such as transcranial magnetic stimulation (TMS) or transcranial direct current stimulation (tDCS) in the rehabilitation of neglect. We found 12 studies including 172 patients (10 TMS studies and 2 tDCS studies) fulfilling our search criteria. Activity of daily living measures such as the Barthel Index or, more specifically for neglect, the Catherine Bergego Scale were the outcome measure in three studies. Five studies were randomized controlled trials with a follow-up time after intervention of up to 6 weeks. One TMS study fulfilled criteria for Class I and one for Class III evidence. The studies are heterogeneous concerning their methodology, outcome measures, and stimulation parameters making firm comparisons and conclusions difficult. Overall, there are however promising results for theta-burst stimulation, suggesting that TMS is a powerful add-on therapy in the rehabilitation of neglect patients.

Keywords: review, rehabilitation, unilateral neglect, transcranial magnetic stimulation, theta-burst protocol, transcranial direct current stimulation

\section{INTRODUCTION}

Hemispatial neglect is a common neurological syndrome that may be particularly disabling after stroke. It is defined as the failure to detect, respond, or orient to the stimuli located in the portion of space contralateral to the lesion (Heilman et al., 1993). Neglect is common, occurring in up to $43 \%$ of patients suffering from an acute right-hemispheric stroke (Ringman et al., 2004). Depending on the assessment, the reported incidence may widely vary between 10 and $82 \%$ following right-hemispheric damage and between 15 and 65\% following left-hemispheric damage (Plummer et al., 2003). Neglect patients show slower functional progress during rehabilitation and need longer hospitalization (Cherney et al., 2001; Gillen et al., 2005). Furthermore, neglect is an independent predictor of poor outcome, in terms of more limited functional independence (Stone et al., 1992; Di Monaco et al., 2011) and lower likelihood of being discharged home (Wee and Hopman, 2005, 2008).

Different therapeutic strategies to treat neglect have been evaluated, such as visual scanning, prism adaptation, sensory stimulation, neck muscle vibration, optokinetic stimulation, or pharmacologic treatments (see for a review Bowen et al., 2002; Kerkhoff and Schenk, 2012). Although these treatments attenuate the severity of neglect, they are often difficult to apply in rehabilitation - particularly during the acute or subacute phase of stroke - due to short duration of effects, patient discomfort, or the difficulty for patients to cooperate (Fierro et al., 2006).

\section{THE CONCEPT OF INTERHEMISPHERIC RIVALRY IN NEGLECT}

The concept of interhemispheric rivalry, based on the model by Kinsbourne $(1987,1993)$, is so far the most common basis for the application of non-invasive brain stimulation (NIBS) to modulate neglect [newer promising approaches are however also thinkable, such as, e.g., rhythmic transcranial magnetic stimulation (TMS) (see Thut et al., 2011) or network modulations (see van der Werf et al., 2010)]. According to this concept, both parietal cortices exert reciprocal interhemispheric inhibition. A damage of the right parietal cortex causes disinhibition of the intact, left hemisphere, and thus a pathological over-activation of the latter. This over-activation in the left, intact hemisphere further depresses the neural activity by an increased inhibition on the damaged hemisphere, aggravating the rightward, ipsilesional attentional bias.

Evidence supporting this concept comes from several experimental approaches. First, seminal works in animal models (Sprague, 1966) and a large body of subsequent studies (see, e.g., Payne and Rushmore, 2004; Rushmore et al., 2006; ValeroCabré et al., 2006) showed that: (a) unilateral interventions (such as lesion, cooling, or TMS) generally introduce an imbalance in the physiological activity between the networks controlling visuospatial attention in the two hemispheres, favoring the intact hemisphere and leading to neglect; and (b) the experimental cancelation of this imbalance (and of neglect) is achievable through the reduction of the hyperexcitability (by lesion or cooling) of specific cortical or subcortical regions in the intact hemisphere. Second, fMRI studies showed a relative hyperactivity of the left, 
undamaged hemisphere in neglect patients, which correlated with neglect severity as measured by behavioral tasks (Corbetta et al., 2005). Moreover, the recovery of neglect correlated with the restoration and rebalancing of activity between both hemispheres, particularly in the dorsal parietal cortex (Corbetta et al., 2005; He et al., 2007). Third, clinical observations also indicate the relevance of the rebalancing of the activity between the two hemispheres as a functional mechanism accompanying neglect recovery. Vuilleumier et al. (1996) described the case of a patient who suffered from two sequential strokes. The first, right-hemispheric stroke, involving the parietal cortex, induced severe neglect, which completely recovered after a second, left-hemispheric stroke involving the frontal eye field. Fourth and finally, the pathological hyperactivity of intact, contralesional areas in neglect patients has also been directly demonstrated by means of a twin-coil TMS approach, allowing to assess the cortical excitability within parieto-motor circuits of the left hemisphere (Koch et al., 2008, 2012). Results showed a significantly higher excitability in neglect patients as compared to healthy controls and to patients with righthemispheric lesions but no neglect. The degree of overexcitability was significantly correlated with neglect severity as measured by paper-pencil tests. Moreover, the application of inhibitory repetitive TMS (rTMS) over the left, contralesional posterior parietal cortex (PPC) could significantly reduce its overexcitability and triggered a significant amelioration in the behavioral measures of neglect.

The results illustrated above thus support the idea that the reinstatement of interhemispheric inhibitory balance is an important mechanism in neglect recovery.

\section{NON-INVASIVE BRAIN STIMULATION}

Non-invasive brain stimulation, i.e., TMS or transcranial direct current stimulation (tDCS), has been increasingly used to interfere with brain activity in healthy subjects and patients with brain lesions. Depending on the stimulation parameters, it is possible to facilitate or to suppress brain activity with measurable behavioral effects.

Transcranial magnetic stimulation is based on the application of very short-lasting, strong electric currents delivered through a coil generating a rapidly changing, high-intensity magnetic field. This magnetic field induces on its part perpendicular currents in the brain, which are strong enough to directly depolarize neurons and influence cortical excitability. rTMS can either enhance $(5-20 \mathrm{~Hz}$, so-called high-frequency stimulation) or suppress ( $\leq 1 \mathrm{~Hz}$, low-frequency stimulation) cortical activity and modulate excitability beyond the duration of the applied stimulation (see for a review Hallett, 2007).

More recently, the so-called "theta-burst stimulation" (TBS) has been introduced as a new protocol. Originally, such protocols were used to induce long-term potentiation (LTP) or long-term depression (LTD) in brain slices (Larson et al., 1986; Abraham, 2003). The protocol consists of three short trains of repetitive highfrequency TMS $(30-100 \mathrm{~Hz})$ in theta-frequency range $(4-7 \mathrm{~Hz})$. The stimulation pattern can have either excitatory (intermittent theta-burst, iTBS) or inhibitory (continuous theta-burst, cTBS) effects on brain activity (Huang et al., 2005). TMS can be used in a variety of ways to induce plastic changes in the brain. An effective way to modulate synaptic efficacy is to activate a cell with two or more inputs at brief intervals, such as in the bursts of the theta-burst protocol. A steady increase in synaptic strength is called LTP, a decrease LTD. In analogy, Huang et al. (2005) developed a modified TBS protocol with a pattern consisting of bursts of three pulses at $50 \mathrm{~Hz}$, repeated every $200 \mathrm{~ms}$ intervals (i.e., at $5 \mathrm{~Hz}$ ). The stimulation intensity was $80 \%$ of the activated motor threshold and the total number of pulses was 600 . They found that a short and intermittent application of TBS (iTBS) facilitated motor-evoked potentials, i.e., increased their amplitude, whereas a continuous application of TBS (cTBS) suppressed motor-evoked potentials for up to $1 \mathrm{~h}$. Nyffeler et al. (2006a, 2009) showed that such LTD-like effects could be disproportionately prolonged by repeated TBS application both in healthy subjects and in patients with neglect. They used a further modified theta-burst protocol with a burst frequency of $30 \mathrm{~Hz}$, repeated with an interburst interval of $100 \mathrm{~ms}$. The stimulation intensity was $80 \%$ of the resting motor threshold, and the total number of pulses was 801. The behavioral outcome was measured in healthy subjects with an oculomotor paradigm. The modified cTBS protocol has been shown to yield conspicuously longer inhibitory effects on the oculomotor cortex [i.e., the frontal eye field, in a head-to-head comparison with the commonly applied $1-\mathrm{Hz}$ stimulation protocol (Nyffeler et al., 2006b)]. Moreover, Nyffeler et al. (2006a, 2009) showed that the behavioral effect of cTBS could be disproportionally prolonged: the behavioral effect after one, two, or four cTBS trains lasted on average up to $30 \mathrm{~min}, 3 \mathrm{~h}$, or $11 \mathrm{~h}$, respectively (Nyffeler et al., 2006a). Similar prolonged behavioral effects after repeated cTBS application were also found in patients with neglect. In a visual perception task, two cTBS trains significantly increased the number of perceived left visual targets for up to $8 \mathrm{~h}$, whereas the application of four cTBS trains significantly increased the number of perceived left targets up to $32 \mathrm{~h}$. No significant improvement was found after sham stimulation (Nyffeler et al., 2009).

While rTMS can generate strong currents capable to depolarize neurons, tDCS changes cortical activity by means of small electric currents. Suggested as a purely neuromodulatory approach, tDCS seems to alter brain activity by influencing the resting membrane potential, and does not evoke action potentials (Fregni and Pascual-Leone, 2007; Nitsche et al., 2008; Paulus, 2011). During tDCS, small currents (1-2 mA) are delivered to the brain transcranially via two large electrodes. The duration of the stimulation, its strength, and its polarity determine the excitability changes. Anodal tDCS leads to excitation of the brain, whereas cathodal tDCS results in brain inhibition (Nitsche and Paulus, 2000). tDCS effects seem to be mainly mediated by changes in the excitability of inhibitory or facilitatory interneuronal circuits that can outlast stimulation duration. tDCS has the advantage that the device is inexpensive, portable, and easy to use, in particular simultaneously with treatment sessions in the rehabilitation setting. Finally, the tingling sensation on the scalp at the beginning of the stimulation fades away shortly after. This is an advantage for a reliable sham condition (i.e., the device can be set to turn off a few seconds after the stimulation beginning, without the subject or the experimenter noticing it), and is also an important element for double-blind, controlled clinical trials. 
The aim of the present study is to review the literature concerning the effectiveness of NIBS in the treatment of neglect patients.

\section{METHODS}

We searched the following databases for studies published in English: PubMed, PsychINFO, and Science Direct. Following search terms were used: neglect, visual neglect, unilateral neglect, rehabilitation, TMS, tDCS. Studies were included in the review if they satisfied following criteria: use of an offline TMS protocol, or use of an online or offline tDCS protocol; treatment of neglect or evaluation of the duration of NIBS effects on neglect as a goal of the study.

\section{RESULTS}

The characteristics of the included studies are presented in Tables 1 and 2 . We found 10 studies that used TMS for neglect rehabilitation, and only 2 studies that used tDCS. In these studies, a total of 172 patients were involved, 147 patients in TMS studies and 25 patients in tDCS studies. The number of included patients varied considerably between studies, from 2 (Shindo et al., 2006) to 27 patients (Kim et al., 2013).

The methodological differences in the rTMS protocols between the studies were also considerable. Five studies used low-frequency rTMS (Brighina et al., 2003; Shindo et al., 2006; Koch et al., 2008; Song et al., 2009; Lim et al., 2010), with frequencies of 0.5, 0.9, or $1 \mathrm{~Hz}$. Three studies used cTBS (Nyffeler et al., 2009; Cazzoli et al., 2012; Koch et al., 2012) with either 30 or $50 \mathrm{~Hz}$ bursts. Finally, two studies (Kim et al., 2010, 2013) compared the effects of low-frequency $(1 \mathrm{~Hz})$ stimulation over the contralesional, intact hemisphere with those of high-frequency rTMS $(20 \mathrm{~Hz})$ over the ipsilesional hemisphere.

Further differences included the number of applied pulses, the duration of the intervention and of the observation period after the intervention, the type of coil used, and the procedure used to determine the stimulation location. The number of TMS pulses varied between 450 (Song et al., 2009) and 1200 pulses per session (Kim et al., 2010, 2013), the cumulative number was between 600 (Koch et al., 2008) and 12,600 pulses (Song et al., 2009). The intervention duration varied between a single session (Koch et al., 2008; Nyffeler et al., 2009; Kim et al., 2010) and 14 sessions (Song et al., 2009).

All studies used a focal, figure-of-eight coil, with the exception of Nyffeler et al. (2009) and Cazzoli et al. (2012), who used a round coil.

Concerning the location of the stimulation site, only one study used a neuronavigation system (Koch et al., 2012). They targeted the left PPC, using individual anatomic MRI and positioning the coil over the angular gyrus close to the posterior part of the adjoining intraparietal sulcus. All other studies used the international 10-20 EEG System. Two studies stimulated over P5 (Brighina et al., 2003; Shindo et al., 2006), all other studies over P3 (or, respectively, P4 for the two studies that entailed ipsilesional stimulation; Kim et al., 2010, 2013).

Five studies were sham-controlled (Nyffeler et al., 2009; Kim et al., 2010, 2013; Cazzoli et al., 2012; Koch et al., 2012), the remaining studies had no sham control group. A control group of patients without neglect was included in three studies (Koch et al., 2008;
Song et al., 2009; Lim et al., 2010). One study (Koch et al., 2012) fulfilled the criteria for Class III evidence, one study (Cazzoli et al., 2012) the criteria for Class I evidence.

In only one study (Brighina et al., 2003) patients had no rehabilitation therapy during the observation. The patients in Lim's study (Lim et al., 2010) received behavioral therapy, and the patients in Koch's study (Koch et al., 2012) received 20 sessions of 45 min therapy. In the remaining four studies (Shindo et al., 2006; Song et al., 2009; Cazzoli et al., 2012; Kim et al., 2013), the patients received a full neurorehabilitation program, including occupational therapy, physiotherapy, and neuropsychology.

The time between brain damage and inclusion varied also considerably between studies. Patients in the acute/subacute stage (first 3 months after brain damage) were included in the studies by Song et al. (2009), Koch et al. (2012), Cazzoli et al. (2012), and Kim et al. (2013). Patients with chronic neglect (more than 3 months after brain damage) were included in the studies by Brighina et al. (2003), Shindo et al. (2006), and Kim et al. (2010). The remaining studies included both patients in the subacute or in the chronic stage.

The follow-up time of the observation of the stimulation effects ranged from 3 days (Nyffeler et al., 2009), 2 weeks (Brighina et al., 2003; Song et al., 2009; Koch et al., 2012), 3 weeks (Cazzoli et al., 2012) to 6 weeks (Shindo et al., 2006). In all studies, no information is provided about a potential fade-out of the stimulation effects over time.

\section{DISCUSSION}

Our database search resulted in 12 studies fulfilling the inclusion criteria. The studies are heterogeneous concerning methodology, evaluation, patients, and post-stroke inclusion time, making firm conclusions about the efficacy of NIBS difficult. In the last few years, at least five reviews (Fierro et al., 2006; Cazzoli et al., 2010; Hesse et al., 2011; Oliveri, 2011; Mylius et al., 2012) specifically addressed the application of TMS or tDCS for the treatment of neglect, and at least another 11 more general reviews (Dobkin, 2004; Rossi and Rossini, 2004; Miniussi et al., 2008; Schlaug and Renga, 2008; Marshall, 2009; Bashir et al., 2010; Langhorne et al., 2011; Miniussi and Rossini, 2011; Stuss, 2011; Vallar and Bolognini, 2011; Schulz et al., 2013) included the topic of brain stimulation in neglect. The number of reviews emphasizes the great interest in the development and establishment of new and current NIBS approaches for the treatment of neglect in particular, and for cognitive rehabilitation in general. However, the mismatch between the number of reviews and the number of original studies represents a compelling call for further systematic investigations in this field.

We found 10 studies using rTMS, and only 2 studies using tDCS. All rTMS studies used inhibitory protocols (low-frequency stimulation or cTBS) and stimulated the contralesional parietal cortex. Two studies (Kim et al., 2010, 2013) also included a condition in which the ipsilesional parietal cortex was stimulated using a highfrequency, excitatory protocol. Nine studies showed a significant improvement after inhibitory stimulation of the contralesional parietal cortex, one study (Kim et al., 2013) found a significant improvement only after ipsilesional excitatory stimulation. The number of patients included in the studies varied between 2 and 27 patients. Four studies evaluated only immediate effects after 


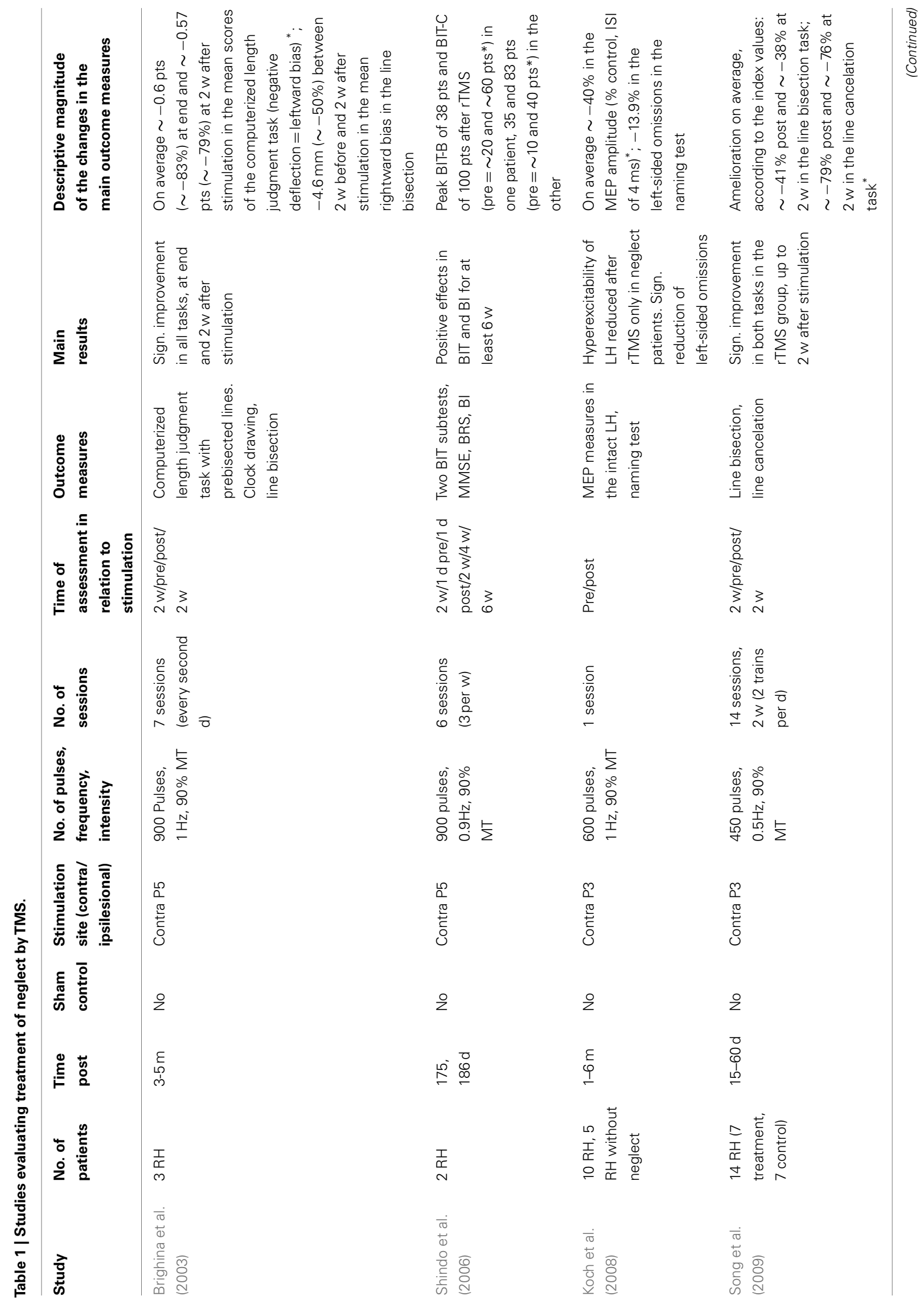




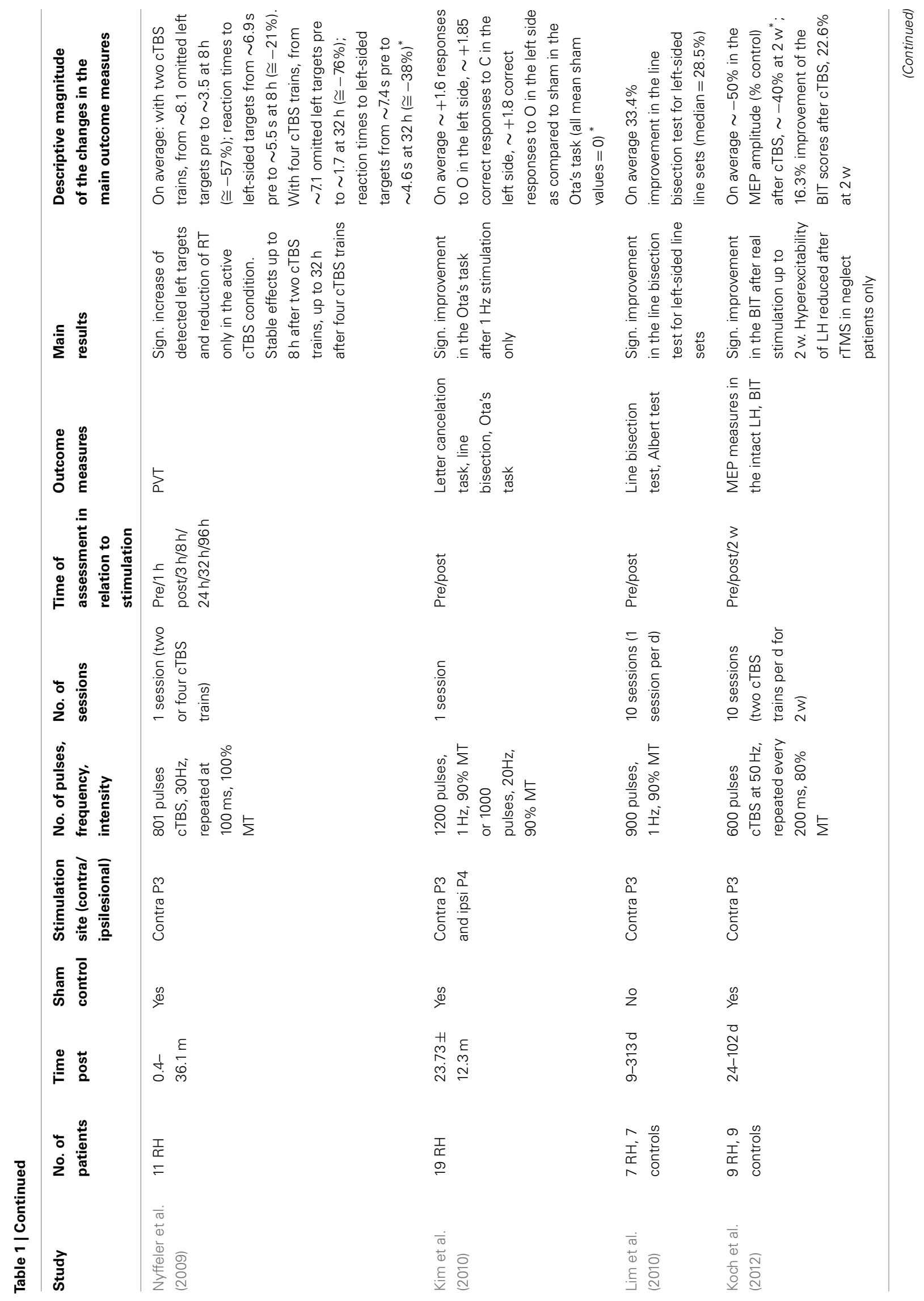




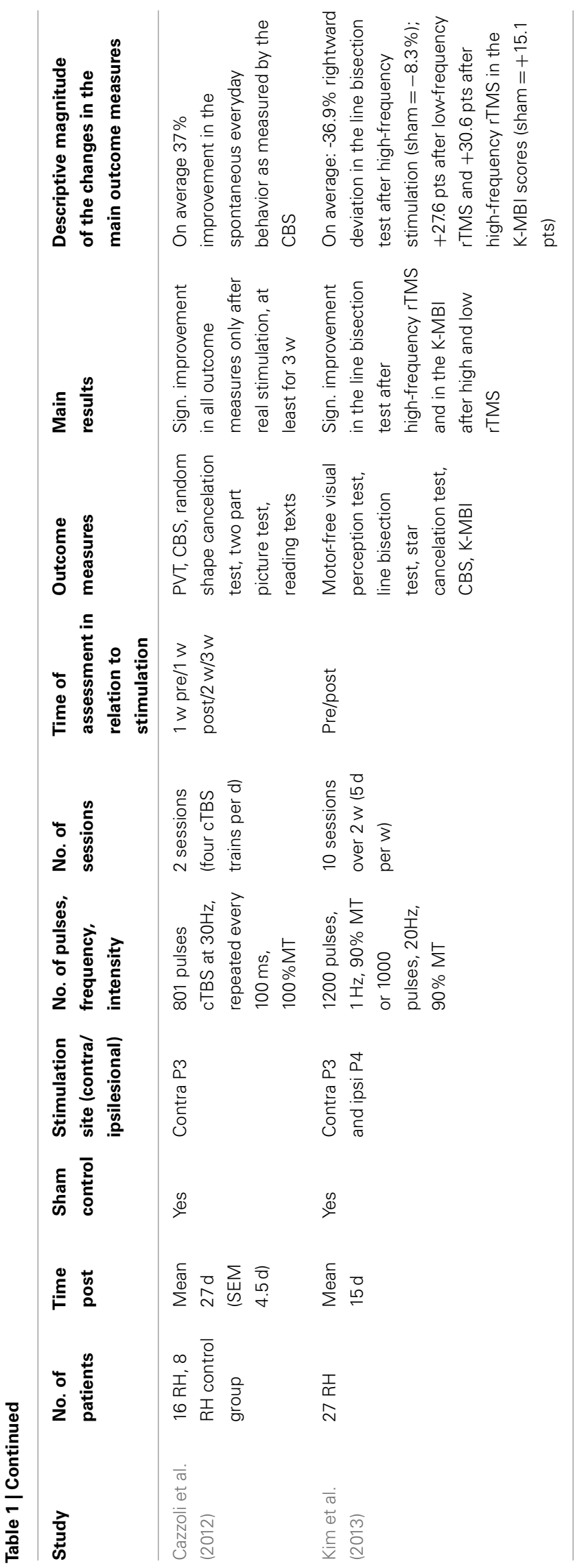

stimulation, without any follow-up measurements. The remaining six studies performed follow-up examinations up to 6 weeks. Five studies were not sham-controlled and, in three studies, activities of daily living (ADL) were evaluated in addition to neuropsychological testing. One study (Cazzoli et al., 2010) fulfilled Class I evidence, and one study (Koch et al., 2012) Class III evidence. In both studies, cTBS of the contralesional parietal cortex was applied.

To the best of our knowledge, no study so far directly compared the different forms of NIBS (e.g., TMS, tDCS) in order to demonstrate the superiority of one method. Both techniques present advantages and disadvantages, and the preference for the application of one technique or the other may also largely depend on the experimental questions and design (see Priori et al., 2009). Moreover, the application of TMS and tDCS should not be seen as mutually exclusive. The combination of the two techniques has in fact been shown to yield promising results, e.g., applying preconditioning by means of tDCS followed by rTMS application (Siebner et al., 2004).

In summary, notwithstanding the limited number of studies, the current state of the evidence looks more promising concerning the studies using cTBS. In the following, we will discuss methodological key points for the future development of treatment concepts of neglect by NIBS.

\section{DIFFERENT EFFECTS OF NIBS ON OUTCOME VARIABLES}

In all studies, a battery of different neuropsychological tests, or test batteries specifically developed for neglect assessment (such as the behavioral inattention test, BIT) were used. Effects of stimulation were often strikingly different across outcome variables, suggesting possible dissociations. One explanation may be methodological: 8 out of the 10 rTMS studies used a focal figure-of-eight coil. Since neglect is associated with multiple lesion sites (e.g., Verdon et al., 2010; Corbetta and Shulman, 2011), a focal stimulation may not be sufficient to improve all aspects tapped by the different neuropsychological tests. It is noteworthy that Cazzoli et al. (2012), who used a non-focal round coil, found significant improvements in all tests. Thus, high focal precision may not be a primary goal for therapeutic rTMS application. However, further studies are needed to evaluate whether focal or non-focal rTMS stimulation of the network involved in neglect has a better clinical outcome.

Three studies also evaluated the effect of TMS on the ADL using the Barthel Index or the Catherine Bergego Scale. Shindo et al. (2006) used the Barthel Index and found a significant improvement after stimulation. Cazzoli et al. (2012) used the Catherine Bergego Scale and also found a significant improvement after real stimulation, but not after sham stimulation. Finally, Kim et al. (2013) used both the Barthel Index and the Catherine Bergego Scale and found a significant improvement only in the Barthel Index.

\section{STIMULATION PROTOCOLS}

Generally, inhibitory stimulation protocols are predominantly applied. Low-frequency $(0.5-1 \mathrm{~Hz})$ repetitive stimulation was used in seven studies. The total number of pulses and daily application varied considerably between studies. The stimulation strength more consistently used was $90 \%$ of the motor threshold. Three 


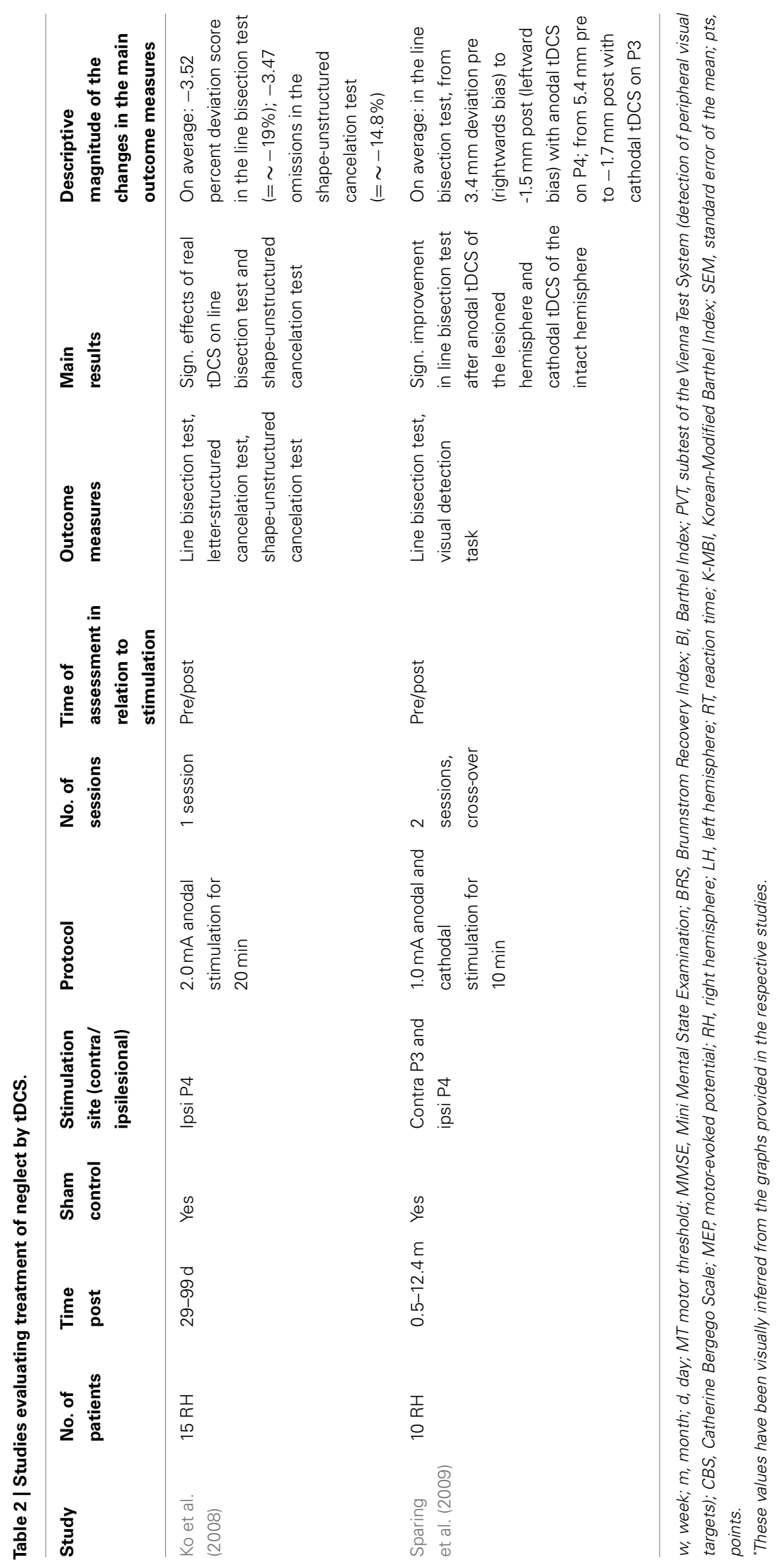


studies used continuous inhibitory cTBS, one study (Koch et al., 2012) used the standard protocol described by Huang et al. (2005), two studies (Nyffeler et al., 2009; Cazzoli et al., 2012) the modified protocol described by Nyffeler et al. (2006a). The two protocols differ in the frequency of the bursts ( 50 versus $30 \mathrm{~Hz}$ ), in the total number of pulses ( 600 versus 801 pulses), and in the definition of the stimulation strength ( $80 \%$ active motor threshold versus $100 \%$ resting motor threshold).

These two protocols were recently compared by Goldsworthy et al. (2012). They stimulated the human primary motor cortex in healthy subjects and recorded motor evoked-potentials (MEP) from the right first dorsal interosseous muscle before and at 0,5 , 10,20 , and $30 \mathrm{~min}$ after stimulation. The results showed that the standard protocol with $50 \mathrm{~Hz}$ induced a neuroplastic response that was short-lived and highly variable, whereas the modified protocol with $30 \mathrm{~Hz}$ induced a lasting change in MEP amplitude that was consistent between subjects. Such a lasting and consistent effect of cTBS may be an advantage for the therapeutic stimulation application. Furthermore, the fact that the repeated cTBS application at the same day can disproportionately prolong its effects (Nyffeler et al., 2009) is a further advantage.

\section{TBS - THE WAY TO AN "IDEAL" STIMULATION PROTOCOL?}

From a clinical and practical point of view, future stimulation protocols for therapeutical interventions should have the following properties: (1) the application should be easy to

\section{REFERENCES}

Abraham, W. C. (2003). How long will long-term potentiation last? Philos. Trans. R. Soc. Lond. B Biol. Sci. 358, 735-744. doi:10.1098/rstb.2002.1222

Bashir, S., Mizrahi, I., Weaver, K., Fregni, F., and Pascual-Leone, A. (2010). Assessment and modulation of neural plasticity in rehabilitation with transcranial magnetic stimulation. $P M R$ 2, S253-S268. doi:10.1016/j.pmrj.2010.10.015

Bowen, A., Lincoln, N. B., and Dewey, M. E. (2002). Spatial neglect: is rehabilitation effective? Stroke 33, 2728-2729. doi:10.1161/01.STR.0000035747. 03607.1A

Brighina, F., Bisiach, E., Oliveri, M., Piazza, A., La Bua, V., Daniele, O., et al. (2003). $1 \mathrm{~Hz}$ repetitive transcranial magnetic stimulation of the unaffected hemisphere ameliorates contralesional visuospatial neglect in humans. Neurosci. Lett. 336, 131-133. doi:10.1016/S03043940(02)01283-1

Cazzoli, D., Müri, R. M., Hess, C. W., and Nyffeler, T. (2010). Treatment of hemispatial neglect by means of rTMS - a review. Restor. Neurol. Neurosci. 28, 499-510. doi:10.3233/RNN-2010-0560

Cazzoli, D., Müri, R. M., Schumacher, R., von Arx, S., Chaves, S., Gutbrod,

perform, i.e., no additional examinations such as neuroimaging or neuronavigation systems should be needed to localize the stimulation site. Indeed, only one study (Koch et al., 2012) used neuronavigation to localize the target site. The remaining studies localized the stimulation site by using the international 10-20 system. (2) The application time should be short. Protocols such as low-frequency stimulation protocols, with daily applications over several weeks, are difficult to perform in a rehabilitation clinic, and are often not well tolerated by patients. In contrast, cTBS application lasts about $40 \mathrm{~s}$. Using the potential of disproportionate prolongation of the effects by repeated cTBS application, Cazzoli et al. (2012) could show that eight cTBS trains applied on 2 days have an ADL-relevant effect of up to 3 weeks.

\section{CONCLUSION}

Our update and review of recent studies using NIBS for neglect treatment shows an ongoing evolution of TMS application from proof-of-concept studies to clinical application. However, the limited number of studies indicates the need of further systematic investigations in this field, with the aim of developing and establishing the most promising stimulation parameters. For cTBS, two recent Class I and III studies demonstrated its clinical utility as add-on therapy in neglect treatment. For tDCS application in neglect, only two studies were found, indicating that this technique may still be in an earlier stage in the evolution toward clinical application.

N. Y. Acad. Sci. 1038, 148-170 doi:10.1196/annals.1315.024

Fierro, B., Brighina, F., and Bisiach, E. (2006). Improving neglect by TMS. Behav. Neurol. 17, 169-176.

Fregni, F., and Pascual-Leone, A. (2007) Technology insight: noninvasive brain stimulation in neurologyperspectives on the therapeutic potential of rTMS and tDCS. Nat. Clin. Pract. Neurol. 3, 383-393. doi:10.1038/ncpneuro0530

Gillen, R., Tennen, H., and McKee, T. (2005). Unilateral spatial neglect: relation to rehabilitation outcomes in patients with right hemisphere stroke. Arch. Phys. Med. Rehabil. 86, 763-767. doi:10.1016/j.apmr.2004.10.029

Goldsworthy, M. R., Pitcher, J. B., and Ridding, M. C. (2012). A comparison of two different continuous theta burst stimulation paradigms applied to the human primary motor cortex. Clin. Neurophysiol. 123, 2256-2263. doi:10.1016/j.clinph.2012.05.001

Hallett, M. (2007). Transcranial magnetic stimulation: a primer. Neuron 55, 187-199. doi:10.1016/j.neuron.2007.06.026

He, B. J., Snyder, A. Z., Vincent, J. L., Epstein, A., Shulman, G. L., and Corbetta, M. (2007). Breakdown of functional connectivity in frontoparietal networks underlies behavioral deficits in spatial neglect. Neuron 53, 905-918. doi:10.1016/j.neuron.2007.02.013

Heilman, K. M., Watson, R. T., and Valenstein, E. (1993). "Neglect and related disorders," in Clinical $\mathrm{Neu}$ ropsychology, eds K. M. Heilman and E. Valenstein (New York: Oxford University Press), 243-294.

Hesse, M. D., Sparing, R., and Fink, G. R. (2011). Ameliorating spatial neglect with non-invasive brain stimulation: from pathophysiological concepts to novel treatment strategies. Neuropsychol. Rehabil. 21, 676-702. doi:10.1080/09602011. 2011.573931

Huang, Y.-Z., Edwards, M. J., Rounis, E., Bhatia, K. P., and Rothwell, J. C. (2005). Theta burst stimulation of the human motor cortex. Neuron 452, 201-206. doi:10.1016/j.neuron.2004.12.033

Kerkhoff, G., and Schenk, T. (2012). Rehabilitation of neglect: an update. Neuropsychologia 50, 1072-1079. doi:10.1016/j

Kim, B. R., Chun, M. H., Kim, D. Y., and Lee, S. J. (2013). Effect of high- and low-frequency repetitive transcranial magnetic stimulation on visuospatial neglect in patients with acute stroke: a doubleblind, sham-controlled trial. Arch. Phys. Med. Rehabil. 94, 803-807. doi:10.1016/j.apmr.2012.12.016 
Kim, J. S., Kim, J. C., Shin, S. H., and Kim, Y. K. (2010). Comparison of effects of repetitive transcranial magnetic stimulation with high- or low-frequency on visuospatial neglect in stroke patients. J. Korean Acad. Rehabil. Med. 34, 397-402.

Kinsbourne, M. (1987). "Mechanisms of unilateral neglect," in Neurophysiological and Neuropsychological Aspects of Spatial Neglect, ed. M. Jeannerod (Amsterdam: Elsevier), 69-86.

Kinsbourne, M. (1993). "Orientational bias model of unilateral neglect: evidence from attentional gradients within hemispace," in Unilateral Neglect: Clinical and Experimental Studies, eds I. H. Robertson and J. C. Marshall (Hove: Lawrence Erlbaum Associates), 63-86.

Ko, M.-H., Han, S.-H., Park, S.H., Seo, J.-H., and Kim, Y.-H. (2008). Improvement of visual scanning after DC brain polarization of parietal cortex in stroke patients with spatial neglect. Neurosci. Lett. 4482, 171-174. doi:10.1016/j.neulet.2008.10.050

Koch, G., Bonní, S., Giacobbe, V., Bucchi, G., Basile, B., Lupo, F., et al. (2012). $\theta$-burst stimulation of the left hemisphere accelerates recovery of hemispatial neglect. Neurology 78, 24-30. doi:10. 1212/WNL.0b013e31823ed08f

Koch, G., Oliveri, M., Cheeran, B., Ruge, D., Lo Gerfo, E., Salerno, S., et al. (2008). Hyperexcitability of parietal-motor functional connections in the intact lefthemisphere of patients with neglect. Brain 131, 3147-3155. doi:10.1093/brain/awn273

Langhorne, P., Bernhardt, J., and Kwakkel, G. (2011). Stroke rehabilitation. Lancet 377, 1693-1702. doi:10.1016/ S0140-6736(11)60325-5

Larson, J., Wong, D., and Lynch, G. (1986). Patterned stimulation at the theta frequency is optimal or the induction of hippocampal long-term potentiation. Brain Res. 368, 347-350. doi:10.1016/00068993(86)90579-2

Lim, J. Y., Kang, E. K., and Paik, N.-J. (2010). Repetitive transcranial magnetic stimulation to hemispatial neglect in patients after stroke: an open-label pilot study. J. Rehabil. Med. 425, 447-452. doi:10.2340/16501977-0553

Marshall, R. S. (2009). Rehabilitation approaches to hemineglect. Neurologist 15, 185-192. doi:10. 1097/NRL.0b013e3181942894
Miniussi, C., Cappa, S. F., Cohen, L. G., Floel, A., Fregni, F., Nitsche, M. A., et al. (2008). Efficacy of repetitive transcranial magnetic stimulation/transcranial direct current stimulation in cognitive neurorehabilitation. Brain Stimul. 1, 326-336. doi:10.1016/j.brs.2008.07.002

Miniussi, C., and Rossini, P. M. (2011). Transcranial magnetic stimulation in cognitive rehabilitation. Neuropsychol. Rehabil. 21, 579-601. doi:10.1080/ 09602011.2011 .562689

Mylius, V., Ayache, S. S., Zouari, H. G., Aoun-Sebaitti, M., Farhat, W. H., and Lefaucheur, J. P. (2012). Stroke rehabilitation using noninvasive cortical stimulation: hemispatial neglect. Expert Rev. Neurother. 12, 983-991. doi:10.1586/ern.12.76

Nitsche, M. A., Cohen, L. G., Wassermann, E. M., Priori, A., Lang, N., Antal, A., et al. (2008). Transcranial direct current stimulation: state of the art 2008. Brain Stimul. 1, 206-223. doi:10.1016/j.brs.2008.06.004

Nitsche, M. A., and Paulus, W. (2000). Excitability changes induced in the human motor cortex by weak transcranial direct current stimulation. J. Physiol. (Lond.) 527, 633-639. doi:10.1111/j.14697793.2000.t01-1-00633.x

Nyffeler, T., Cazzoli, D., Hess, C. W., and Müri, R. M. (2009). One session of repeated parietal theta burst stimulation trains induces long-lasting improvement of visual neglect. Stroke 408, 2791-2796. doi:10.1161/STROKEAHA.109. 552323

Nyffeler, T., Wurtz, P., Lüscher, H. R., Hess, C. W., Senn, W., Pflugshaupt, T., et al. (2006a). Extending lifetime of plastic changes in the human brain. Eur. J. Neurosci. 24, 2961-2966. doi:10.1111/j.14609568.2006.05154.x

Nyffeler, T., Wurtz, P., Lüscher, H. R., Hess, C. W., Senn, W., Pflugshaupt, T., et al. (2006b). Repetitive TMS over the human oculomotor cortex: comparison of $1-\mathrm{Hz}$ and theta burst stimulation. Neurosci. Lett. 409, 57-60. doi:10.1016/j.neulet.2006.09.011

Oliveri, M. (2011). Brain stimulation procedures for treatment of contralesional spatial neglect. Restor. Neurol. Neurosci. 29, 421-425. doi:10.3233/RNN-2011-0613

Paulus, W. (2011). Transcranial electrical stimulation (tES - tDCS; tRNS, tACS) methods. Neuropsychol. Rehabil. 21, 602-617. doi:10.1080/09602011
Payne, B. R., and Rushmore, R. J. (2004). Functional circuitry underlying natural and interventional cancellation of visual neglect. Exp. Brain Res. 154, 127-153. doi:10.1007/s00221-003-1660-9

Plummer, P., Morris, M. E., and Dunai, J. (2003). Assessment of unilateral neglect. Phys. Ther. 83, 732-740.

Priori, A., Hallett, M., and Rothwell, J. C. (2009). Repetitive transcranial magnetic stimulation or transcranial direct current stimulation? Brain Stimul. 2, 241-245. doi:10.1016/j.brs Ringman, J. M., Saver, J. L., Woolson, R. F., Clarke, W. R., and Adams, H. P. (2004). Frequency, risk factors, anatomy, and course of unilateral neglect in an acute stroke cohort. Neurology 63, 468-474 doi:10.1212/01.WNL.0000133011. 10689.CE

Rossi, S., and Rossini, P. M. (2004). TMS in cognitive plasticity and the potential for rehabilitation. Trends Cogn. Sci. 8, 273-279. doi:10.1016/j.tics.2004.04.012

Rushmore, R. J., Valero-Cabre, A., Lomber, S. G., Hilgetag, C. C., and Payne, B. R. (2006). Functional circuitry underlying visual neglect. Brain 129, 1803-1821. doi:10.1093/brain/awl140

Schlaug, G., and Renga, V. (2008). Transcranial direct current stimulation: a noninvasive too to facilitate stroke recovery. Expert Rev. Med. Devices 5, 759-768. doi:10.1586/17434440.5.6.759

Schulz, R., Gerloff, C., and Hummel, F. C. (2013). Non-invasive brain stimulation in neurological diseases. Neuropharmacology 64, 579-587. doi:10.1016/j.neuropharm.2012. 05.016

Shindo, K., Sugiyama, K., Huabao, L., Nishijima, K., Kondo, T., and Izumi, S. (2006). Long-term effect of low-frequency repetitive transcranial magnetic stimulation over the unaffected posterior parietal cortex in patients with unilateral spatial neglect. J. Rehabil. Med. 38, 65-67. doi:10.1080/16501970500441807

Siebner, H. R., Lang, N., Rizzo, V., Nitsche, M. A., Paulus, W., Lemon, R. N., et al. (2004). Preconditioning of low-frequency repetitive transcranial magnetic stimulation with transcranial direct current stimulation: evidence for homeostatic plasticity in the human motor cortex. J. Neurosci. 24, 3379-3385. doi:10.1523/JNEUROSCI.531603.2004

Song, W., Du, B., Xu, Q., Hu, J., Wang, M., and Luo, Y. (2009).
Low-frequency transcranial magnetic stimulation for visual spatial neglect: a pilot study. J. Rehabil. Med. 413, 162-165. doi:10.2340/16501977-0302

Sparing, R., Thimm, M., Hesse, M. D., Küst, J., Karbe, H., and Fink, G. R. (2009). Bidirectional alterations of interhemispheric parietal balance by non-invasive cortical stimulation. Brain 132, 3011-3020. doi:10.1093/brain/ awp154

Sprague, J. M. (1966). Interaction of cortex and superior colliculus in mediation of visually guided behavior in the cat. Science 153, 1544-1547. doi:10.1126/science.153.3743.1544

Stone, S. P., Patel, P., Greenwood, R. J., and Halligan, P. W. (1992). Measuring visual neglect in acute stroke and predicting its recovery: the visual neglect recovery index. J. Neurol. Neurosurg. Psychiatr. 55, 431-436. doi:10.1136/jnnp.55.6.431

Stuss, D. T. (2011). The future of cognitive neurorehabilitation. Neuropsychol. Rehabil. 21, 755-768. doi:10.1080/09602011. 2011.605590

Thut, G., Veniero, D., Romei, V., Miniussi, C., Schyns, P., and Gross, J. (2011). Rhythmic TMS causes local entrainment of natural oscillatory signatures. Curr. Biol. 21, 1176-1185. doi:10.1016/j.cub.2011.05.049

Valero-Cabré, A., Rushmore, R. J., and Payne, B. R. (2006). Low frequency transcranial magnetic stimulation on the posterior parietal cortex induces visuotopically specific neglect-like syndrome. Exp. Brain Res. 172, 14-21. doi:10.1007/s00221005-0307-4

Vallar, G., and Bolognini, N. (2011). Behavioural facilitation following brain stimulation: implications for neurorehabilitation. Neuropsychol. Rehabil. 21, 618-649. doi:10.1080/09602011.2011. 574050

van der Werf, Y. D., Sanz-Arigita, E. J., Menning, S., and van den Heuvel, O. A. (2010). Modulating spontaneous brain activity using repetitive transcranial magnetic stimulation. $B M C$ Neurosci. 11:145. doi:10.1186/14712202-11-145

Verdon, V., Schwartz, S., Lovblad, K. O., Hauert, C. A., and Vuilleumier, P. (2010). Neuroanatomy of hemispatial neglect and its functional components: a study using voxel-based lesion-symptom mapping. Brain 133, 880-894. doi:10.1093/brain/ awp305 
Vuilleumier, P., Hester, D., Assal, G., and Regli, F. (1996). Unilateral spatial neglect recovery after sequential strokes. Neurology 46, 184-189. doi:10.1212/WNL.46.1.184

Wee, J. Y., and Hopman, W. M. (2005). Stroke impairment predictors of discharge function, length of stay, and discharge destination in stroke rehabilitation. Am. J. Phys. Med. Rehabil. 84, 604-612. doi:10. 1097/01.phm.0000171005.08744.ab
Wee, J. Y., and Hopman, W. M. (2008). Comparing consequences of right and left unilateral neglect in a stroke rehabilitation population. Am. J. Phys. Med. Rehabil. 87, 910-920. doi:10.1097/PHM.0b013e31818a $58 \mathrm{bd}$

Conflict of Interest Statement: The authors declare that the research was conducted in the absence of any commercial or financial relationships that could be construed as a potential conflict of interest.

Received: 12 March 2013; accepted: 19 May 2013; published online: 10 June 2013.

Citation: Müri RM, Cazzoli D, Nef T, Mosimann UP, Hopfner $S$ and Nyffeler T (2013) Non-invasive brain stimulation in neglect rehabilitation: an update. Front. Hum. Neurosci. 7:248. doi: 10.3389/fnhum.2013.00248

Copyright (C) 2013 Müri, Cazzoli, Nef, Mosimann, Hopfner and Nyffeler. This is an open-access article distributed under the terms of the Creative Commons Attribution License, which permits use, distribution and reproduction in other forums, provided the original authors and source are credited and subject to any copyright notices concerning any third-party graphics etc. 